\title{
Expression of autophagy-related genes in cerebrospinal fluid of patients with tuberculous meningitis
}

\author{
YUNBO MA ${ }^{1}$, YONGXIA ZHANG $^{2}$, YANYAN ZHAO ${ }^{3}$, XIANHUA WANG $^{4}$, \\ YUEFU LIN ${ }^{1}$ and AIGUO MA ${ }^{4}$ \\ Departments of ${ }^{1}$ Preventive Medicine, ${ }^{2}$ Emergency and ${ }^{3}$ Clinical Laboratory, Linyi People's Hospital, Linyi, \\ Shandong 276034; ${ }^{4}$ School of Public Health, Qingdao University, Qingdao, Shandong 266021, P.R. China
}

Received August 21, 2017; Accepted December 11, 2017

DOI: $10.3892 /$ etm.2018.5999

\begin{abstract}
The expression of autophagy-related genes in cerebrospinal fluid of patients with tuberculous meningitis (TBM) and their clinical significance in patients with TBM was investigated. Sixty patients with TBM (observation group) and twenty healthy volunteers during the same period (control group) were selected and the cerebrospinal fluid was collected. The expression levels of p62, Beclin1 and LC3-II genes in cerebrospinal fluid were detected via semi-quantitative reverse transcription-polymerase chain reaction and patients in observation group were divided into high expression and normal or low expression group on the basis of LC3-II expression levels. On the other hand, the contents of inflammatory factors interleukin-6, -10 (IL-6, -10), and tumor necrosis factor- $\alpha$ (TNF- $\alpha$ ) were detected using the enzyme-linked immunosorbent assay kit. The mRNA levels of p62, Beclin1 and LC3-II in cerebrospinal fluid of patients in observation were significantly higher than those in the control group $(\mathrm{P}<0.01)$. TUNEL assay showed that the apoptosis level of cerebro-spinal fluid in high expression was obviously lower than that in normal or low expression group $(\mathrm{P}<0.01)$; the content of IL-6 and TNF- $\alpha$ in cerebrospinal fluid in high expression was significantly lower than those in normal or low expression group $(\mathrm{P}<0.01)$; the content of IL-10 in cerebrospinal fluid in high expression was obviously higher than that in normal or low expression group $(\mathrm{P}<0.01)$. Correlation analysis revealed that LC3-II was positively correlated with IL-10, but negatively correlated with IL-6 and TNF- $\alpha$. The mRNA levels of p62, Beclin1 and LC3-II in cerebrospinal fluid of patients with TBM are increased, there is a correlation between expression levels of autophagy-related genes and inflammatory factors, and the high expression of
\end{abstract}

Correspondence to: Dr Yuefu Lin, Department of Preventive Medicine, Linyi People's Hospital, 233 Fenghuang Street, Hedong, Linyi, Shandong 276034, P.R. China

E-mail: linyuefuly@126.com

Key words: autophagy interleukin-10, LC3-II, tuberculous meningitis autophagy-related genes may have a protective effect on patients with TBM.

\section{Introduction}

Tuberculous meningitis (TBM) is an infectious disease of the central nervous system caused by autologous Mycobacterium tuberculosis infection, which can affect the spinal cord in severe cases, thus resulting in high non-cure rate of the disease $(1,2)$. The early clinical symptoms of TBM are not obvious, and the patients often cannot receive timely treatment due to misdiagnosis and errors, thereby missing the best treatment time (3). At present, the clinical treatment method of TBM is mainly drug therapy, but the commonly-used drugs will lead to obvious drug resistance, and thus to significantly poor prognosis and even death, so it is urgent to search for new therapeutic target drugs (4). Ní Cheallaigh et al (5) found that the autophagy response in cerebrospinal fluid in meningitis patients is in an activated state, and the activation of autophagy can reduce the damage of inflammatory response to the body and produce a certain protective effect. Autophagy is a kind of catabolic process involving lysosomes, which is a bond that links the non-specific immune system and specific immune system of the body, and plays an important role in the infection control and immune homeostasis maintenance. The autophagy response will affect the expression levels of degradation substrate p62, autophagy constitutive protein LC3-II and autophagy-associated protein Beclin1 (6-8). At present, there has been no research on the relationship between TBM and autophagy, and the correlation of autophagy activation with TBM-induced inflammatory response remains unclear. The present study aimed to assist the new mechanism of TBM and provide new clinical treatment considerations.

\section{Patients and methods}

Objects. A total of 60 patients, including 28 males and 32 females aged 42-63 years, treated and diagnosed as TBM in Linyi People's Hospital (Linyi, China) from March, 2014 to March, 2015 were enrolled in the study, while normal volunteers, including 10 males and 10 females aged $40-60$ years, in the same age group were the controls. The general clinical manifestations of the TBM patients enrolled were fever, 
headache, nausea and vomiting; patients were diagnosed via cerebrospinal fluid, imaging and many other clinical indexes according to the international expert diagnosis of tuberculosis in 2010, and suspicious cases were excluded. Patients with other tuberculosis infections, other consumptive diseases or a past history of central nervous system diseases or malignant tumors were eliminated; all the patients enrolled signed the informed consent and all clinicopathologic data and treatment programs were saved. The present study was approved by the Ethics Committee of Linyi People's Hospital.

Instruments and materials. TRIzol kit and Prime Script RT Reagent kit (both from Invitrogen; Thermo Fisher Scientific, Inc., Waltham, MA, USA); rabbit monoclonal anti-p62 (cat. no. 23214), rabbit monoclonal anti-Beclin1 (cat. no. 3495), rabbit monoclonal anti-LC3-II (cat. no. 3868), rabbit monoclonal anti-glyceraldehyde-3-phosphate dehydrogenase (GAPDH) (cat. no. 5174) and horseradish peroxidase-labeled rabbit secondary monoclonal antibody (cat. no. 7074), (dilution, 1:100) (all from Cell Signaling Technology, Inc., Danvers, MA, USA) electrochemiluminescence liquid, and developing powder (both from Invitrogen, Thermo Fisher Scientific, Inc.), human interleukin-6 (IL-6) enzyme-linked immunosorbent assay (ELISA) kit, human IL-10 ELISA kit and human tumor necrosis factor- $\alpha$ (TNF- $\alpha$ ) ELISA kit (all from Boster Biological Technology, Pleasanton, CA, USA); TUNEL kit (Nanjing Jiancheng Bioengineering Institute, Nanjing, China); polymerase chain reaction (PCR) instrument (ABI USA, Vernon, CA, USA); ultraviolet imaging system (Biometra GmbH, Göttingen, Germany); other relevant equipment and reagents are described in the relevant parts.

Grouping. A total of 60 TBM patients were selected as the observation group, while 20 normal volunteers in the same age group were selected as the control group. The general clinical data of observation and control group were analyzed, and the results showed that the differences in age and sex between the two groups were not statistically significant $(\mathrm{P}>0.05)$.

Extraction of cerebrospinal fluid. The cerebrospinal fluid was obtained from patients and volunteers via lumbar puncture (9): Under the lateral position, the puncture site was disinfected and covered with surgical towel, followed by puncture with a puncture needle to extract $5 \mathrm{ml}$ fresh cerebrospinal fluid; then the cerebrospinal fluid was stored at $-80^{\circ} \mathrm{C}$ for standby application.

Semi-quantitative RT-PCR detection. Fresh cerebrospinal fluid $(0.5 \mathrm{ml})$ was taken and added with $1 \mathrm{ml}$ pre-cooled TRIzol. After standing in ice bath for $5 \mathrm{~min}, 1 \mathrm{ml}$ chloroform was added and vibrated for $15 \mathrm{sec}$. After standing for $3 \mathrm{~min}$, the mixture was centrifuged at $4^{\circ} \mathrm{C}$ at $9,000 \mathrm{x}$ g for $10 \mathrm{~min}$. The supernatant was transferred, and the same volume of isopropyl alcohol was added and vibrated evenly. After standing for $15 \mathrm{~min}$, the mixture was centrifuged at $4^{\circ} \mathrm{C}$ at $9,000 \mathrm{x} \mathrm{g}$ for $10 \mathrm{~min}$. Then the supernatant was discarded, $75 \%$ ethanol was slowly added along the tube wall and manually turned upside down to wash 5 times, followed by centrifugation at $4^{\circ} \mathrm{C}$ for $2 \mathrm{~min}$ at $10,800 \mathrm{x}$. The above operation was repeated twice
Table I. PCR procedures.

\begin{tabular}{lcc}
\hline $\begin{array}{l}\text { Reaction } \\
\text { temperature }\end{array}$ & Reaction time & Cycles \\
\hline $95^{\circ} \mathrm{C}$ & $5 \mathrm{~min}$ & \\
$95^{\circ} \mathrm{C}$ & $30 \mathrm{sec}$ & 30 cycles \\
$59^{\circ} \mathrm{C}$ & $40 \mathrm{sec}$ & \\
$72^{\circ} \mathrm{C}$ & $1 \mathrm{~min}$ & \\
$72^{\circ} \mathrm{C}$ & $5 \mathrm{~min}$ & \\
\hline
\end{tabular}

PCR, polymerase chain reaction.

Table II. PCR primers.

\begin{tabular}{ll}
\hline Genes & \multicolumn{1}{c}{ Sequence } \\
\hline p62 & F: 5'-TGCCCCTCTTCTGTCTCATAGT-3' \\
& R: 5'-CACTTGTTTTGCTGCCCTAAAT-3' \\
Beclin1 & F: 5'-CTGGGGACCTTTTTGACATC-3' \\
& R: 5'-TTGCGGTTCTTTTCCACGTC-3' \\
LC3-II & F: 5'-AATCCCGGTGATAATAGAAC-3' \\
& R: 5'-TTTCATCCCGAACGTCTCC-3' \\
GAPDH & F:5'-TGAAGGTCGGAGTCAACGGATTTGGT-3' \\
& R: 5'-AAATGAGCCCCAGCCTTCTCCATG-3'
\end{tabular}

GAPDH, glyceraldehyde-3-phosphate dehydrogenase; PCR, polymerase chain reaction.

and then the ethanol was discarded. The cover of centrifuge tube was opened and the ethanol evaporated naturally. After that, $80 \mu \mathrm{l}$ diethyl pyrocarbonate (DEPC) water was added to dissolve RNA, and then $2 \mu \mathrm{l}$ mixture was taken and diluted with $98 \mu 1$ DEPC water. The concentration of RNA and A260/A280 were measured using the nucleic acid protein quantometer. Whether RNA was degraded was detected via agarose gel electrophoresis. The results revealed that RNA in each group was of higher quality and could be used for subsequent experiments. A total of $4 \mu 15 x$ Prime Script Buffer, $1 \mu 1$ Prime Script RT Enzyme MixI and $1 \mu$ l OligodT Primer were added and the RNA was added until the total volume was $800 \mathrm{ng}$ according to the concentration of RNA. Then RNase-free water was added to prepare it into the $20 \mu \mathrm{l}$ system, followed by reverse transcription at $37^{\circ} \mathrm{C}$ for $15 \mathrm{~min}$ and $85^{\circ} \mathrm{C}$ for $5 \mathrm{sec}$. After cDNA was taken, the primer and PCR Master Mix were added in proportion for PCR according to the conditions shown in Table I. The primer sequences are shown in Table II. After that, the expression level of each gene was detected via $2 \%$ agarose gel electrophoresis with GAPDH as the internal reference. The expression levels of different genes in each group were determined by p62/GAPDH, Beclin1/GAPDH and LC3-II/GAPDH, respectively.

Detection of apoptosis in cerebrospinal fluid via TUNEL. The fresh cerebrospinal fluid was added into the 6-well plate with slides, added with $4 \%$ paraformaldehyde and fixed at $4^{\circ} \mathrm{C}$ for 
A

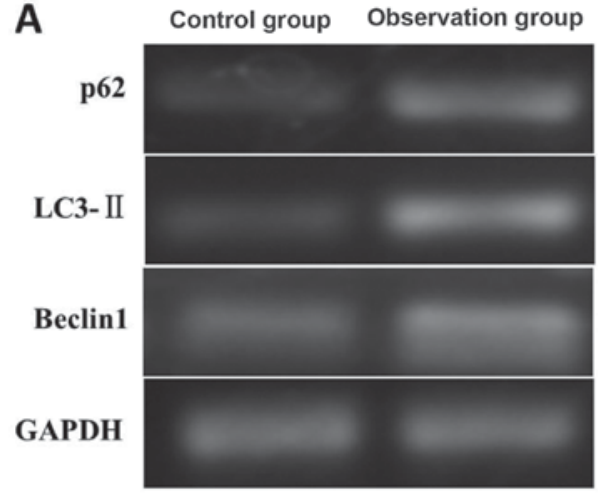

C

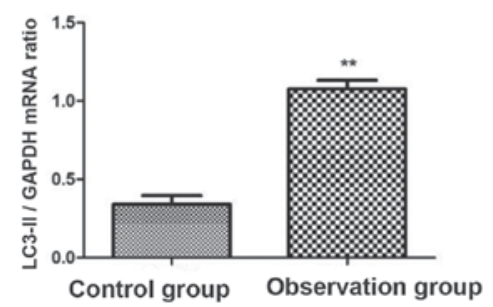

B

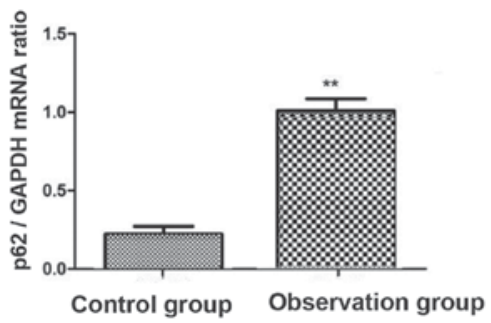

D

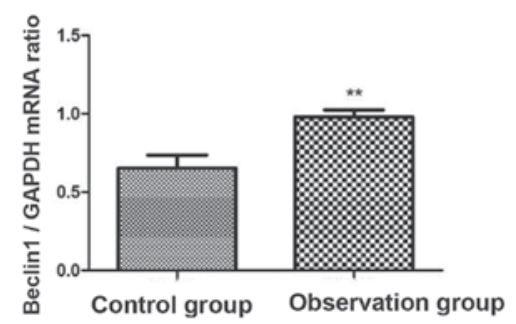

Figure 1. Expression levels of autophagy-related genes in cerebrospinal fluid in TBM patients. (A) Agarose gel electropherogram. (B) Relative expression level of p62. (C) Relative expression level of LC3-II. (D) Relative expression level of Beclin1. The mRNA levels of p62, Beclin1 and LC3-II in cerebrospinal fluid in control group are significantly lower than those in observation group, ${ }^{* *} \mathrm{P}<0.01$. TBM, tuberculous meningitis.

30 min. After fixation, it was washed with pre-cooled phosphate buffered saline (PBS) 3 times ( 5 min/each time). FJ-B dye solution in the TUNEL kit was diluted and added into the treated samples in each group, followed by incubation at $4^{\circ} \mathrm{C}$ for $90 \mathrm{~min}$ and washing with PBS. Finally, the samples were sealed with DAPI glycerin, observed and photographed under an inverted fluorescence microscope (Olympus Corporation, Tokyo, Japan).

Detection of inflammatory factors in cerebrospinal fluid via ELISA. The standard solution in IL-6, -10 and TNF- $\alpha$ ELISA kits was diluted to $800,600,400,300,200$ and $100 \mathrm{pg} / \mathrm{ml}$ for determination of standard curve. The biotin-labeled antibodies were diluted 100 times to prepare it into the working solution. After $100 \mu \mathrm{l}$ sample or standard substance was added into each well, the plate was sealed for reaction at $37^{\circ} \mathrm{C}$ for $60 \mathrm{~min}$. After the reaction was over, IL-6, -10 and TNF- $\alpha$ antibodies were added following strictly the instructions of the kit for reaction for $90 \mathrm{~min}$. After the reaction was over, $300 \mu \mathrm{l}$ buffer was added. After standing for $1 \mathrm{~min}$, the plate was patted dry and washed 5 times. Then $100 \mu 1$ avidin-peroxidase compound was added, followed by plate sealing and reaction at $37^{\circ} \mathrm{C}$ for $60 \mathrm{~min}$. The OD value at the wavelength of $450 \mathrm{~nm}$ of each well was measured after the stop buffer was added. Finally, the sample concentration of each group was analyzed and calculated using CurveExpert 1.5 software.

Statistical analysis. The data in the present study are presented as mean \pm standard deviation (SD) and SPSS 19.0 software (SPSS Inc., Chicago, IL, USA) was used for data processing. A t-test was used for measurement data. Analysis of variance was used for comparison among groups, t-test was used for comparison of means between the two groups, and Pearson's test was used for correlation of factors. $\mathrm{P}<0.05$ was considered to indicate a statistically significant diference.

\section{Results}

Expression of autophagy-related genes. The expression levels of p62, Beclin1 and LC3-II in cerebrospinal fluid of patients in observation group and control group were detected via semi-quantitative reverse transcription-polymerase chain reaction (RT-PCR). The results showed that the mRNA levels of p62, Beclin1 and LC3-II in observation group were significantly higher than those in control group, and the differences were statistically significant $(\mathrm{P}<0.01)$. Patients in observation group were divided based on the mRNA expression level of LC3-II: Patients with the mRNA expression level of LC3-II lower or equal to that in control group as the low or normal expression group $(n=22)$, and those with the mRNA expression level of LC3-II higher than that in control group as the high expression group $(\mathrm{n}=38)$ (Fig. 1).

Detection of cell apoptosis in cerebrospinal fluid via TUNEL. Cell apoptosis in cerebrospinal fluid in high expression group and low or normal expression group was detected using the TUNEL kit. The results showed that the number of apoptotic cells in cerebrospinal fluid in high expression group was significantly lower than that in low or normal expression groups (Fig. 2).

Levels of inflammatory factors in cerebrospinal fluid. The levels of inflammatory factors in cerebrospinal fluid in high expression group and low or normal expression group were detected using the ELISA kit. The results revealed that the level of IL-10 in cerebrospinal fluid in high expression group 


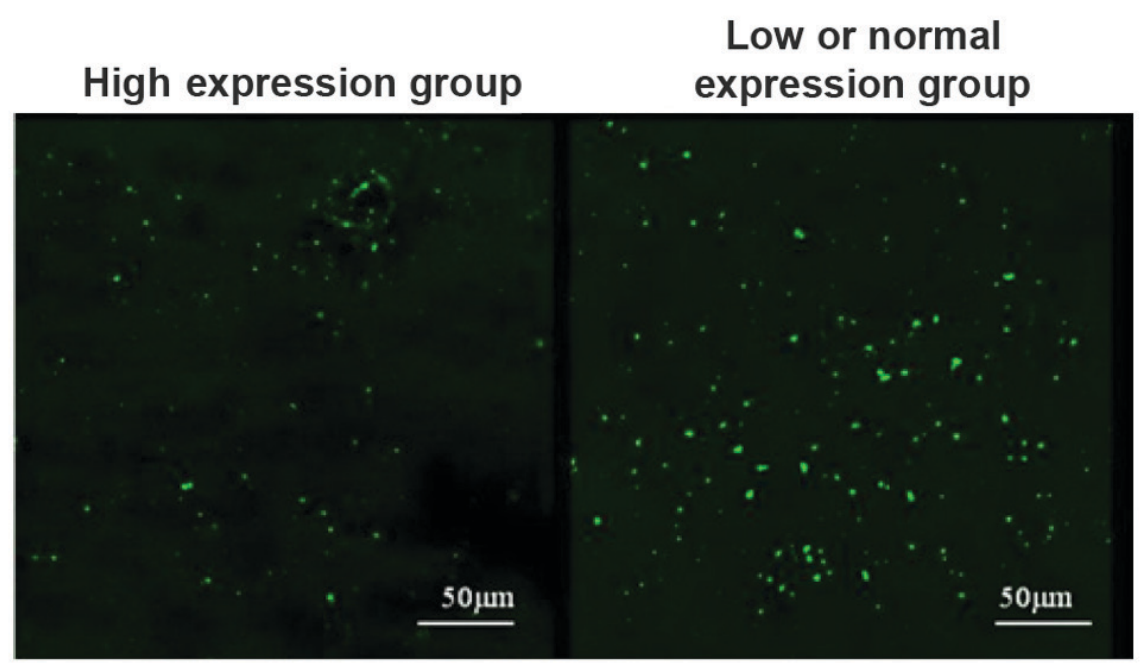

Figure 2. Cell apoptosis in cerebrospinal fluid in TBM patients. The number of apoptotic cells in cerebrospinal fluid in high expression group is significantly lower than that in low or normal expression group. TBM, tuberculous meningitis.
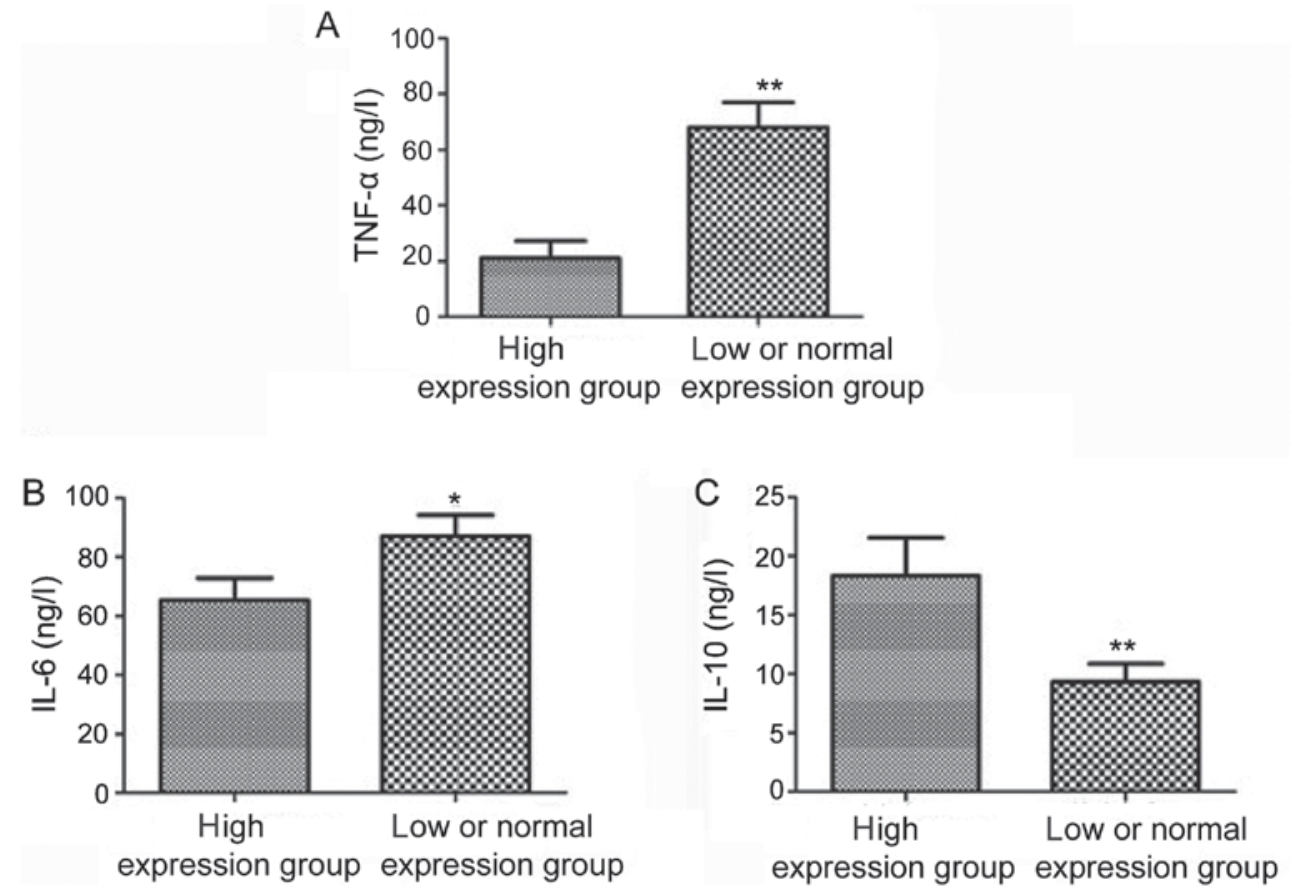

Figure 3. Contents of inflammatory factors in cerebrospinal fluid in TBM patients. (A) Statistical chart of TNF- $\alpha$ content. (B) Statistical chart of IL-6 content (C) Statistical chart of IL-10 content; the levels of IL-6 and TNF- $\alpha$ in high expression group are obviously lower than those in low or normal expression group, but the IL-10 level is obviously higher than that in low or normal expression group. ${ }^{* *} \mathrm{P}<0.01,{ }^{*} \mathrm{P}<0.05$. TBM, tuberculous meningitis; TNF- $\alpha$, tumor necrosis factor- $\alpha$; IL-6 - 10 , interleukin-6 -10.

was obviously higher than that in low or normal expression group, but the levels of IL-6 and TNF- $\alpha$ were obviously lower than those in low or normal expression group; the differences were statistically significant $(\mathrm{P}<0.05, \mathrm{P}<0.01)$ (Fig. 3).

Analysis of correlation between autophagy-related genes and inflammatory factors. The relative expression level of LC3-II mRNA and the contents of IL-6, -10 and TNF- $\alpha$ in cerebrospinal fluid in TBM patients were recorded in detail, followed by correlation analysis. The mRNA level of LC3-II was positively correlated with the content of IL-10 $(r=0.5326, \mathrm{P}<0.05)$ (Fig. 4). The mRNA level of LC3-II was negatively correlated with the content of IL-6 (r=-0.7342, P<0.05) (Fig. 5). Besides, the mRNA level of LC3-II was also negatively correlated with the content of TNF- $\alpha(r=-0.5972, \mathrm{P}<0.05)$ (Fig. 6).

\section{Discussion}

TBM is a central inflammatory disease caused by a single pathogenic bacterium, which can cause death in severe cases. Therefore, the treatment and prevention of TBM is an urgent problem $(10,11)$. Inflammatory factors are small molecule polypeptides that mediate the inflammatory response, which is synthesized and released by the body after stimulation. 


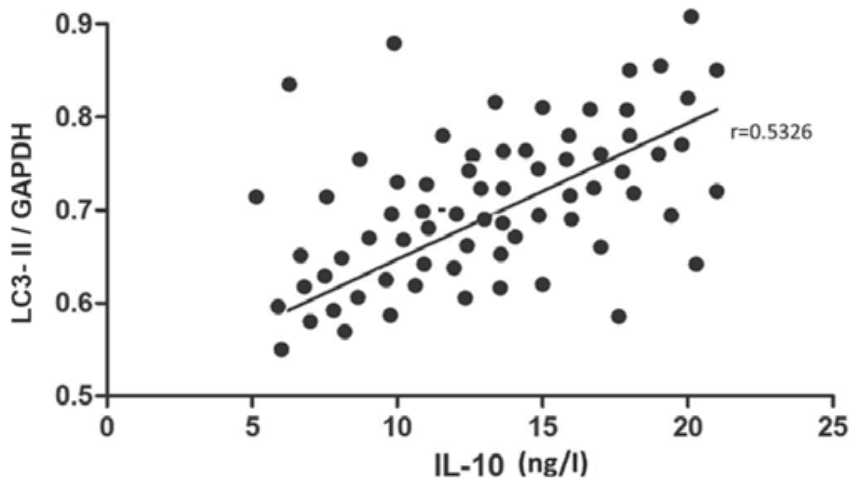

Figure 4. Analysis of correlation between mRNA level of LC3-II and content of IL-10; the mRNA level of LC3-II is positively correlated with the content of IL-10 ( $r=0.5326, \mathrm{P}<0.05)$. IL-10, interleukin-10.

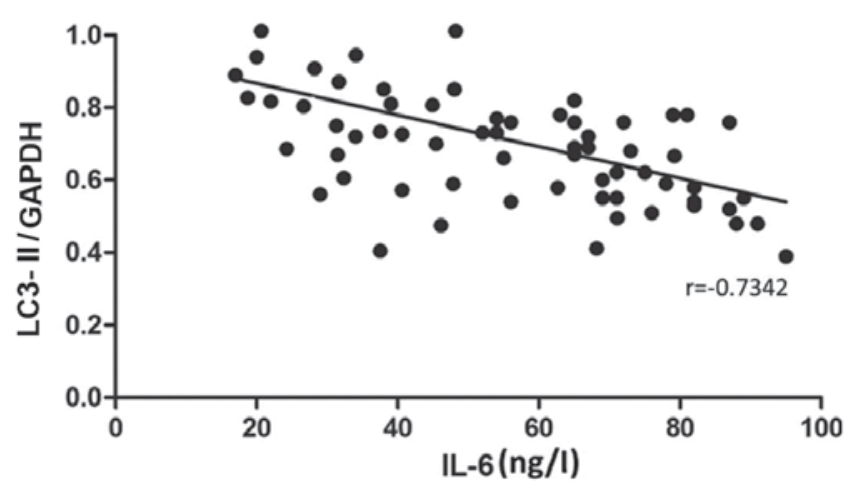

Figure 5. Analysis of correlation between mRNA level of LC3-II and content of IL-6; the mRNA level of LC3-II is negatively correlated with the content of IL-6 $(r=-0.7342, \mathrm{P}<0.05)$. IL-6, interleukin-6.

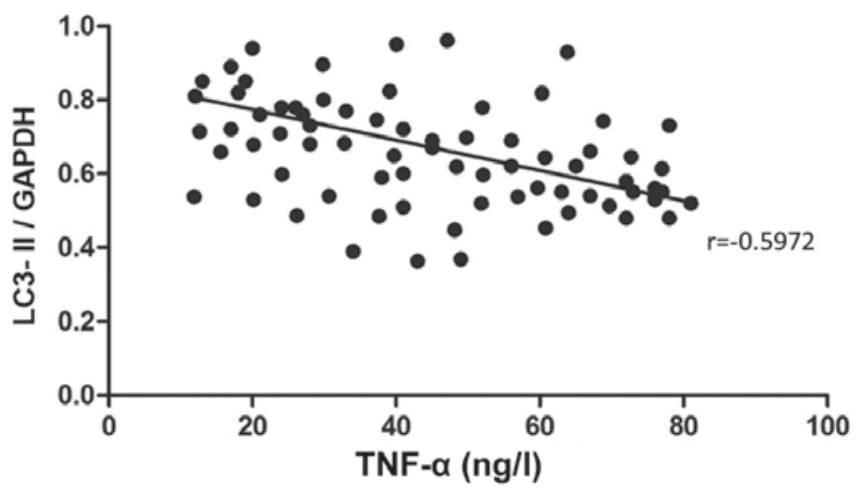

Figure 6. Analysis of correlation between mRNA level of LC3-II and content of TNF- $\alpha$; the mRNA level of LC3-II is negatively correlated with the content of TNF- $\alpha(r=-0.5972, \mathrm{P}<0.05)$. TNF- $\alpha$, tumor necrosis- $\alpha$.

The level of inflammatory factors is closely related to the diagnosis, treatment and prognosis of inflammation $(12,13)$. Marais et al (14) revealed that the levels of inflammatory factors in cerebrospinal fluid in TBM patients are significantly different from those in normal people, so the changes in inflammatory factor levels can be used as auxiliary indexes for TBM diagnosis. The study of Netea-Maier et al (15) found that LPS-induced inflammation in mice can activate autophagy in the body, and autophagy agonists can significantly reduce the increased pro-inflammatory cytokines caused by inflammatory response, reduce the inflammatory response and increase the survival rate of mice to a certain extent. Autophagy is a self-digestive reaction in the body, and moderate autophagy can protect the body. When autophagy is activated, a large number of free membrane structures can be formed, which can wrap the damaged organelles, protein aggregates and other substances to be degraded. With the gradual maturation, it can bind with lysosomes to form autophagic lysosomes; moreover, proteolytic enzymes in lysosomes can effectively degrade its internal components, and reduce the levels of long-lived protein and some cytokines in the body (16). Lapaquette et al (17) found that autophagy is closely related to most cellular stress response pathways, and autophagy affects multiple parts of the immune system and participates in the regulation of inflammatory responses.

In the present study, the expression of autophagy-related genes in cerebrospinal fluid in TBM patients were investigated, the levels of inflammatory factors in cerebrospinal fluid in TBM patients were analyzed and the correlation between contents of inflammatory factors and autophagy-related genes was also analyzed. It was found in the present study that the mRNA expression levels of p62, LC3-II and Beclin1 in cerebrospinal fluid in TBM patients were significantly higher than those in normal people. The above results indicated that autophagy is in an activated state in cerebrospinal fluid in TBM patients and involved in the TBM-induced inflammatory response. Moors et al (18) found that the level of autophagy marker protein LC3-II in the striatum of patients with Parkinson's disease is increased and the autophagic degradation substrate p62 is also increased, which is consistent with the results of this experiment. Studies have found that the chronic inflammation of the nervous system is an important factor of inducing Parkinson's disease; in other words, the inflammatory response can activate autophagy to a certain extent. The levels of inflammatory factors in cerebrospinal fluid in TBM patients were detected via ELISA. The results revealed that the levels of pro-inflammatory cytokines, IL-6 and TNF- $\alpha$, in cerebrospinal fluid in patients with high expression of LC3-II mRNA were significantly lower than those in patients with low or normal expression, but the level of anti-inflammatory factor IL-10 in cerebrospinal fluid in patients with high expression of LC3-II mRNA was significantly increased. The activation of autophagy can reduce the levels of pro-inflammatory cytokines in cerebrospinal fluid in TBM patients through degradation. When the levels of inflammatory factors in cerebrospinal fluid are decreased, the levels of anti-inflammatory factors will be increased to a certain extent under the regulation of negative feedback mechanism, thus further reducing the inflammatory response and protecting the body (19). The study by Nakahira et al (20) found that the relationship between inflammatory response and autophagy is complex, inflammatory stimuli can activate autophagy, and autophagy can further degrade the inflammatory stimuli in the body and reduce the inflammatory response. In this experiment, the correlation between LC3-II mRNA level and inflammatory factor levels was analyzed, and the results revealed that the LC3-II mRNA level was positively correlated with IL-10, but negatively correlated with IL- 6 and TNF- $\alpha$. The above results showed that autophagy is closely related to the inflammatory 
response of TBM, which may treat TBM through mediating the smooth autophagy activity in the body to remove waste accumulated in the body in time under pathological conditions, such as damaged organelles and protein aggregates. However, there were some shortcomings in this study. The mechanism of autophagy-related genes in regulating inflammatory factor levels in cerebrospinal fluid in TBM patients was not deeply studied, but it will be a direction in later research.

In conclusion, autophagy is activated in cerebrospinal fluid in TBM patients, and the mRNA levels of Beclin1 and LC3-II in cerebrospinal fluid are increased, which is correlated with the inflammatory factor levels in cerebrospinal fluid, so it can provide reference for the clinical treatment of TBM.

\section{Acknowledgements}

Not applicable.

\section{Funding}

No funding was received.

\section{Availability of data and materials}

The datasets used and/or analyzed during the current study are available from the corresponding author on reasonable request.

\section{Authors' contributions}

YM wrote the manuscript and participated with statistical analysis. YoZ contributed to conception and design. YaZ helped with the data of TUNEL. XW and YL conducted semi-quantitative RT-PCR and ELISA method. AM interpreted the results. All authors read and approved the final manuscript.

\section{Ethics approval and consent to participate}

The present study was approved by the Ethics Committee of Linyi People's Hospital (Linyi, China). All the patients enrolled signed the written informed consent.

\section{Consent for publication}

Not applicable.

\section{Competing interests}

The authors declare that they have no competing interests.

\section{References}

1. Palomo FS, Rivero MGC, Quiles MG, Pinto FP, Machado AMO and Carlos Campos Pignatari A: Comparison of DNA extraction protocols and molecular targets to diagnose tuberculous meningitis. Tuberc Res Treat 2017: 5089046, 2017.

2. Thwaites G, Caws M, Chau TT, D'Sa A, Lan NT, Huyen MN, Gagneux S, Anh PT, Tho DQ, Torok E, et al: Relationship between Mycobacterium tuberculosis genotype and the clinical phenotype of pulmonary and meningeal tuberculosis. J Clin Microbiol 46: 1363-1368, 2008.
3. Gupta R, Garg RK, Jain A, Malhotra HS, Verma R and Sharma PK: Spinal cord and spinal nerve root involvement (myeloradiculopathy) in tuberculous meningitis. Medicine (Baltimore) 94: e404, 2015.

4. Iype T, Pillai AK, Cherian A, Nujum ZT, Pushpa C, Dae D and Krishnapillai V: Major outcomes of patients with tuberculous meningitis on directly observed thrice a week regime. Ann Indian Acad Neurol 17: 281-286, 2014.

5. Ní Cheallaigh C, Keane J, Lavelle EC, Hope JC and Harris J: Autophagy in the immune response to tuberculosis: Clinical perspectives. Clin Exp Immunol 164: 291-300, 2011.

6. Xie Y, Kang R, Sun X, Zhong M, Huang J, Klionsky DJ and Tang D: Posttranslational modification of autophagy-related proteins in macroautophagy. Autophagy 11: 28-45, 2015.

7. Zhou XJ and Zhang H: Autophagy in immunity: Implications in etiology of autoimmune/autoinflammatory diseases. Autophagy 8: 1286-1299, 2012.

8. Liu Y and Levine B: Autosis and autophagic cell death: The dark side of autophagy. Cell Death Differ 22: 367-376, 2015.

9. Zhao Y, Bu H, Hong K, Yin H, Zou Y-L, Geng S-J, Zheng MM and He JY: Genetic polymorphisms of CCL1 rs2072069 G/A and TLR2 rs3804099 T/C in pulmonary or meningeal tuberculosis patients. Int J Clin Exp Pathol 8: 12608-12620, 2015.

10. Christensen A-SH, Roed C, Omland LH, Andersen PH, Obel N and Andersen $\AA \mathrm{B}$ : Long-term mortality in patients with tuberculous meningitis: A Danish nationwide cohort study. PLoS One 6: 713-725, 2011.

11. Sanei Taheri M, Karimi MA, Haghighatkhah H, Pourghorban R, Samadian M and Delavar Kasmaei H: Central nervous system tuberculosis: An imaging-focused review of a reemerging disease. Radiol Res Pract 2015: 202806, 2015.

12. Robb CT, Regan KH, Dorward DA and Rossi AG: Key mechanisms governing resolution of lung inflammation. Semin Immunopathol 38: 425-448, 2016.

13. Slavich GM, Irwin MR, Einaudi S, Wasniewska M, Miccoli M and Baroncelli GI: From stress to inflammation and major depressive disorder: A social signal transduction theory of depression. Psychol Bull 140: 774-815, 2014.

14. Marais S, Wilkinson KA, Lesosky M, Coussens AK, Deffur A, Pepper DJ, Schutz C, Ismail Z, Meintjes G and Wilkinson RJ: Neutrophil-associated central nervous system inflammation in tuberculous meningitis immune reconstitution inflammatory syndrome. Clin Infect Dis 59: 1638-1647, 2014.

15. Netea-Maier RT, Plantinga TS, van de Veerdonk FL, Smit JW and Netea MG: Modulation of inflammation by autophagy: Consequences for human disease. Autophagy 12: 245-260, 2016.

16. Huber TB, Edelstein CL, Hartleben B, Inoki K, Jiang M, Koya D, Kume S, Lieberthal W, Pallet N, Quiroga A, et al: Emerging role of autophagy in kidney function, diseases and aging. Autophagy 8: 1009-1031, 2012.

17. Lapaquette P, Guzzo J, Bretillon L and Bringer M-A: Cellular and molecular connections between autophagy and inflammation. Mediators Inflamm 2015: 398483, 2015.

18. Moors TE, Hoozemans JJM, Ingrassia A, Beccari T, Parnetti L, Chartier-Harlin M-C and van de Berg WDJ: Therapeutic potential of autophagy-enhancing agents in Parkinson's disease. Mol Neurodegener 12: 266-271, 2017.

19. Chen Z-H, Wu Y-F, Wang P-L, Wu Y-P, Li Z-Y, Zhao Y, Zhou JS, Zhu C, Cao C, Mao YY, et al: Autophagy is essential for ultrafine particle-induced inflammation and mucus hyperproduction in airway epithelium. Autophagy 12: 297-311, 2016.

20. Nakahira K, Cloonan SM, Mizumura K, Choi AM and Ryter SW: Autophagy: A crucial moderator of redox balance, inflammation, and apoptosis in lung disease. Antioxid Redox Signal 20: 474-494, 2014.

This work is licensed under a Creative Commons Attribution-NonCommercial-NoDerivatives 4.0 International (CC BY-NC-ND 4.0) License. 